\title{
Evaluación de tres protocolos hormonales para la inducción de la espermiación en yaque Leiarius marmoratus
}

\section{Evaluation of three protocols for hormonal induction of sper- miation in yaque Leiarius marmoratus}

\author{
Tatiana Mira L,* Zoot, Mauricio Medina R, M.Sc, Pablo Cruz C, Ph.D.
}

Universidad de los Llanos, Instituto de Acuicultura de los Llanos. Grupo de Investigación sobre Reproducción y Toxicología de los Organismos Acuáticos - GRITOX, km 12 vía Puerto López, Villavicencio, Colombia. *Correspondencia: t.mirah@gmail.com

Recibido: Mayo 18 de 2009; Aceptado: Marzo 18 de 2010.

\section{RESUMEN}

Objetivo. Evaluar los efectos de la inducción hormonal con extracto de hipófisis de carpa (EHC) y sGnRHa+domperidona (OVA), sobre la liberación de fluido seminal y sus características. Materiales y métodos. Los tratamientos consistieron en: T1 tres dosis de EHC: $0.2 \mathrm{mg} / \mathrm{kg}$ (Oh), $0.5 \mathrm{mg} / \mathrm{kg}$ (9h) y $2 \mathrm{mg} / \mathrm{kg}(18 \mathrm{~h}) ; \mathrm{T} 2: 8 \mathrm{mg} / \mathrm{kg}$ de EHC (0h), $5 \mathrm{mg}$ de EHC (24h) y $0.5 \mathrm{ml}$ de OVA (48h); T3: dosis única de $0.25 \mathrm{ml}$ de OVA y Control: $3 \mathrm{ml}$ de suero fisiológico en tres inyecciones $(0,24$ y $48 \mathrm{~h})$. Fueron evaluados el volumen, movilidad masal e individual, tiempo de activación, velocidad y concentración espermática mediante análisis semi-cuantitativo y análisis espermático asistido por computador (CASA). Resultados. T1, T2 y T3 mostraron volumen seminal significativamente mayor $(2.7 \pm 0.7 ; 2.6 \pm 0.5$ y $1.8 \pm 0.4 \mathrm{ml})$ comparados con el control $(0.2 \pm 0.1 \mathrm{ml})$. La movilidad y tiempo de activación de T2 y T3 presentaron los mejores resultados ( $95 \pm 0,0 \%$ durante $64.6 \pm 3$ seg y $89.4 \%$ durante 63.6 seg, respectivamente). T3 presentó movilidad total individual más alta $(88.9 \pm 4.5 \%)$ que la observada con T1 $(60.6 \pm 5.6 \%)$, siendo ésta igual a T2 y al control $(68.2 \pm 11.5 \%$ y $81.2 \pm 1.6 \%$, respectivamente). El porcentaje de espermatozoides inmóviles fue mayor en T1 y T2 que en T3 y el control. T3 mostró la mayor velocidad curvilínea y velocidad promedio de desplazamiento $(64.3 \pm 9.2$ y $57.0 \pm 9.6 \mu \mathrm{m} / \mathrm{seg})$. La concentración espermática fue mayor $(p<0.05)$ en T3 y el control $\left(6.255 \pm 1.322\right.$ y 9.460 sptz $\left.\times 10^{3} / \mu \mathrm{l}\right)$. Conclusiones. Los tratamientos hormonales con EHC y OVA, aplicados individualmente o combinados, son efectivos para inducir la espermiación en L. marmoratus.

Palabras clave: Domperidona, extracto de hipófisis de carpa, GnRH, reproducción, semen, siluriformes. 
Mira - Evaluación protocolos hormonales para inducción de la espermiación

\section{ABSTRACT}

Objective. To assess the effects of hormonal induction using pituitary carp extract (PCE) and sGnRHa + domperidone (OVA) on the release of seminal fluid and its characteristics. Materials and methods. Treatments were: T1: PCE administered in three doses : $0.2 \mathrm{mg} /$ $\mathrm{kg}(0 \mathrm{~h}), 0.5 \mathrm{mg} / \mathrm{kg}(9 \mathrm{~h})$ and $2 \mathrm{mg} / \mathrm{kg}(18 \mathrm{~h}), \mathrm{T} 2: 8 \mathrm{mg} / \mathrm{kg}$ of PCE $(0 \mathrm{~h}), 5 \mathrm{mg}$ of PCE (24 h) and $0.5 \mathrm{ml}$ of OVA $(48 \mathrm{~h})$, T3: single dose of $0.25 \mathrm{ml}$ of OVA and Control: $3 \mathrm{ml}$ of saline solution administered in three doses (0, 24 and $48 \mathrm{~h})$. Volume, mass and individual motility, duration of movement, speed and sperm concentration were evaluated using semi-quantitative assessment and Computer Assisted Sperm Analisis (CASA). Results. T1, T2 and T3 showed a significantly greater volume $(2.7 \pm 0.7,2.6 \pm 0.5$ and $1.8 \pm 0.4 \mathrm{ml})$ compared with the control $(0.2 \pm 0.1 \mathrm{ml})$. T2 and T3 showed the best results in motility and duration of movement, ( $95 \pm 0.0 \%$ for $64.6 \pm 3 \mathrm{sec}$ and $89.4 \%$ for $63.6 \mathrm{sec}$ respectively). T3 had the highest individual total motility $(88.9 \pm 4.5 \%)$ in contrast to $T 1(60.6 \pm 5.6 \%)$, and equal to $\mathrm{T} 2$ and the control $(68.2 \pm 11.5 \%$ and $81.2 \pm 1.6 \%)$. The percentage of immotile spermatozoa was higher in T1 and T2 than T3 and the control. T3 showed the greatest curvilinear velocity and average velocity of displacement $(64.3 \pm 9.2$ and $57.0 \pm 9.6 \mu \mathrm{m} / \mathrm{sec})$. The sperm concentration was higher $(p<0.05)$ in T3 and the control $(6255 \pm 1322$ and 9.460sptz $\left.\mathrm{x} 10^{3} / \mathrm{il}\right)$. Conclusions. Hormonal induction with PCE and OVA, applied individually or combined, are effective for spermiation induction in L. marmoratus.

Key words: Domperidone, $\mathrm{GnRH}$, pituitary carp extract, reproduction, semen, siluriformes.

\section{NTRODUCCIÓN}

Muchos peces de interés comercial con potencial para ser introducidos a la acuicultura presentan disfunciones reproductivas cuando son mantenidos en cautiverio. Estas disfunciones probablemente resultan de la combinación del estrés generado por el cautiverio y a la pérdida de las condiciones ambientales apropiadas para su reproducción natural (1). Estas disfunciones reproductivas en los machos se manifiestan como una disminución en el volumen $o$ en la calidad seminal (1). En silúridos como Clarias gariepinus y Leiarius marmoratus, no ocurre liberación de semen bajo condiciones de cautividad, por lo que es necesario sacrificar el macho o utilizar técnicas quirúrgicas para extraer los gametos directamente del testículo $(2,3)$. Diferentes inductores de segunda y tercera generación han sido evaluados para producir liberación y aumento del volumen seminal en varias especies de silúridos como: Sorubim cuspicaudus (4), Pseudoplatystoma fasciatum (5), Rhamdia sebae (6) y Pangasius bocourti (7), siendo los más utilizados extractos hipofisiarios heterólogos de pez (carpa), análogos superactivos de la hormona liberadora de gonadotropinas (sGnRH-A) combinados con bloqueadores de dopamina y gonadotropina coriónica humana, todos con resultados variables. En C. gariepinus, Viveiros et al (2), probaron diferentes protocolos con base en extracto hipofisiario de carpa y de Clarias, mGnRHa con Pimozide y Ovaprim, de manera individual y en varias combinaciones. En los tratamientos donde lograron obtener semen por medio de extrujamiento, este fue de reducida cantidad e inferior calidad cuando se comparó con el semen extraído directamente del testículo (2).

El objetivo de este trabajo fue evaluar los efectos normales sobre la espermiación y la calidad seminal del yaque.

\section{MATERI ALES Y MÉTODOS}

Sitio de estudio. El estudio se realizó en la estación piscícola del Instituto de Acuicultura de la Universidad de los Llanos, localizada a una altitud de $418 \mathrm{~m}$ sobre el nivel del mar, con pluviosidad de $4050 \mathrm{~mm}$ anuales, 
humedad relativa del $75 \%$, temperatura ambiental media de $27^{\circ} \mathrm{C}$ y temperatura promedio del agua de $25^{\circ} \mathrm{C}$.

Material biológico. Se utilizaron 22 machos adultos de $1,41 \pm 0,07 \mathrm{~kg}$ de peso $y$ $55,14 \pm 1,18 \mathrm{~cm}$ longitud total. Los reproductores fueron alojados en estanques de tierra de $372 \mathrm{~m}^{2}$ a una densidad promedio de $204 \mathrm{~g} / \mathrm{m}^{2}$, donde fueron alimentados con concentrado comercial del $25 \%$ de PB, una vez al día en las horas de la tarde, todos los días.

Fueron seleccionados con base en las características de madurez sexual, evidenciada por la emisión de un fluido transparente y oleoso a través de la papila urogenital ante presión ventral Los individuos seleccionados se trasladaron a piletas de cemento con 9,6 $\mathrm{m}^{3}$ de agua, donde permanecieron por lo menos $24 \mathrm{~h}$ antes de iniciar el proceso de inducción y hasta el final del experimento. Allí se identificaron por medio de microchips previamente implantados y se registró el peso $(\mathrm{kg})$ y la longitud $(\mathrm{cm})$ total de cada individuo.

I nducción hormonal. Se evaluaron 3 tratamientos, utilizando extracto hipofisiario de carpa (EHC) y una mezcla comercial de análogo superactivo de hormona liberadora de gonadotropina con un bloqueador de los receptores D2 de dopamina, Ovaprim $\AA$ (OVAP: sGnRHa + domperidona). Los tratamientos consistieron en aplicar vía intramuscular tres inyecciones de $0.2 \mathrm{mg} /$ $\mathrm{Kg}(\mathrm{Oh}), 0.5 \mathrm{mg} / \mathrm{Kg}(9 \mathrm{~h})$ y $2 \mathrm{mg} / \mathrm{Kg}$ (18h) de EHC para el tratamiento 1 (T1) (Recomedación personal); 8mg/kg de EHC (Oh), $5 \mathrm{mg} / \mathrm{kg}$ de EHC (24h) y $0.5 \mathrm{ml} / \mathrm{kg}$ de OVAP (24h) en el segundo tratamiento (T2) (2) y una sola inyección de $0.25 \mathrm{ml} / \mathrm{Kg}$ de OVAP en el tercer tratamiento (Recomendación de la casa fabricante Syndel, Canadá). Los machos control recibieron inyecciones de solución salina fisiológica, siguiendo el mismo cronograma de los individuos tratados.

Durante el experimento se midieron parámetros fisicoquímicos del agua, tales como temperatura, oxígeno disuelto, $\mathrm{pH}$ y conductividad, empleando una sonda multiparamétrica YSI 556 (Yellow Spring Instruments).

Obtención de los gametos. Transcurridas 9h (T1) (recomendación personal), 16h (T2) (2) y $12 \mathrm{~h}$ (T3) (Recomendación casa fabricante) después de aplicada la última dosis se extrajo el fluido seminal. Previo a la extracción de los gametos, los animales fueron tranquilizados por inmersión en una solución de 300ppm de fenoxietanol (Sigma Co, Saint Louis, Missouri). Una vez observados síntomas de anestesia se retiró de la solución, se secó cuidadosamente en el área ventral y se realizó presión manual entre las aletas pectorales y pélvicas en sentido cráneo caudal; el fluido expulsado por la papila urogenital, se colectó en viales aforados tipo Eppendorf® (Brand, Alemania). El fluido extraído fue llevado inmediatamente al laboratorio donde se mantuvo a una temperatura de $28^{\circ} \mathrm{C}$ durante las evaluaciones espermáticas.

Evaluación seminal. Se determinó la concentración espermática mediante una cámara de recuento celular Neubauer. Para evaluar la movilidad masal (MM) y el tiempo de activación espermática (TA) se empleó una gota $(20 \mu \mathrm{l})$ de semen colocada sobre una lámina excavada (profundidad 1- $1.2 \mathrm{~mm}$, Micro-Slides Premiere, China) dispuesta en un microscopio óptico $(10 \times$ de magnificación), la cual se activó con agua corriente $(180 \mu \mathrm{l})$. Desde la activación hasta el momento en que el $90 \%$ de los espermatozoides cesaron el movimiento, se registró el tiempo con un cronómetro. Adicionalmente se determinaron parámetros de movilidad y velocidad espermática por medio de un sistema de análisis espermático asistido por computador (CASA- Computer Assisted Sperm Analisis, Medea Lab, Alemania). Para este procedimiento se colocó $1 \mu \mathrm{l}$ de semen en una cámara de Makler dispuesta en un microscopio óptico con contraste de fase (Nikon E400, Japón), se activó con agua corriente (c.a. $75 \mu$ l, volumen ajustado con la concentración espermática) permitiendo la observación de aproximadamente 200 espermatozoides por campo. Los parámetros evaluados fueron: Movilidad progresiva lineal rápida (MPLR), Movilidad progresiva lineal lenta (MPLL), 
Movilidad local o nado en círculo (MLC), porcentaje de espermatozoides inmóviles (IMV), Movilidad total espermática (MTI: porcentaje de espermatozoides móviles), Movilidad local espermática (MIL), Movilidad circular espermática (MCI), Velocidad curvilínea (VCL: Velocidad real a lo largo de una trayectoria), Velocidad en línea recta (VLR: distancia en línea recta desde el inicio hasta el final del desplazamiento, dividida por el tiempo de duración del desplazamiento) y Velocidad promedio de desplazamiento (VPD).

Análisis de resultados. Los resultados fueron expresados como media \pm error estándar de la media (SEM). Para determinar los efectos de los tratamientos, los resultados fueron sometidos a un análisis de varianza de una vía (ANOVA), previa validación de los supuestos de homogeneidad de varianza y normalidad de los datos, seguido de una prueba de comparación de Tukey-Kramer, con un nivel de significancia de $p<0.05$.

\section{RESULTADOS}

Durante los experimentos se observaron los siguientes parámetros de calidad de agua: temperatura $25.8 \pm 0.18^{\circ} \mathrm{C}$; oxígeno disuelto $5.95 \pm 0.36 \mathrm{mg} / \mathrm{L}$; saturación de OD $76.45 \pm$ $1.85 \%$; conductividad $19 \pm 1.74 \mu \mathrm{s} / \mathrm{cm}$; $\mathrm{pH}$ $6.13 \pm 0.28$ y salinidad $0.01 \pm 0.001 \mathrm{ppm}$.

Macroscópicamente las muestras seminales provenientes de los individuos tratados presentaron coloración blanquecina, mientras que las muestras de los machos control fueron casi trasparentes. La viscosidad fue alta en las muestras seminales obtenidas de los machos del T2, lo cual dificultó su manipulación y evaluación. La tabla 1 muestra las características seminales observadas con los diferentes tratamientos, en los 22 machos estudiados.

En todos los tratamientos, el porcentaje de respuesta a la espermiación fue de $100 \%$. Sin embargo, el volumen seminal obtenido fue significativamente mayor en los machos pertenecientes al T1, cuando fueron comparados con el grupo control (2.84 \pm $0.6 \mathrm{ml}$ y $0.15 \pm 0.1 \mathrm{ml}$, respectivamente);
Tabla 1. Características seminales de yaque, tratados con tres protocolos hormonales para inducir la espermiación. Letras diferentes indican diferencias significativas $(p<0.05)$. Valores mostrados corresponden a la media \pm SEM.

\begin{tabular}{|c|c|c|c|c|c|}
\hline $\mathbf{T}$ & Rta & $\begin{array}{l}\text { Vol } \\
(\mathrm{ml})\end{array}$ & $\begin{array}{l}\text { Mov } \\
(\%)\end{array}$ & $\begin{array}{c}\text { TA } \\
\text { (seg) }\end{array}$ & $\begin{array}{l}\text { CESP } \\
\text { (sptz }\end{array}$ \\
\hline $\mathrm{T} 1$ & 09-Sep & $\begin{array}{l}2.84^{3} \\
\pm 0.6\end{array}$ & $\begin{array}{c}81.3^{\circ 0} \\
\pm 4.4\end{array}$ & $\begin{array}{l}52.7^{3} \\
\pm 5.5\end{array}$ & $\begin{array}{c}1.873^{b} \\
\pm 274\end{array}$ \\
\hline $\mathrm{T} 2$ & 04-Abr & $\begin{array}{c}2.55^{5 b} \\
\pm 0.5\end{array}$ & $\begin{array}{l}95.0^{3} \\
\pm 0.0\end{array}$ & $\begin{array}{l}64.6^{\circ} \\
\pm 3.1\end{array}$ & $\begin{array}{c}2.505^{\circ} \\
\pm 532\end{array}$ \\
\hline T3 & 06-Jun & $\begin{array}{c}1.59^{5 b} \\
\pm 0.4\end{array}$ & $\begin{array}{c}90.8^{3 b} \\
\pm 1.7\end{array}$ & $\begin{array}{l}64.9^{\circ} \\
\pm 2.5\end{array}$ & $\begin{array}{c}6.303^{\mathrm{s}} \\
\pm 1.025\end{array}$ \\
\hline CONT & 3 & $\begin{array}{l}0.15^{\circ} \\
\pm 0.1\end{array}$ & $\begin{array}{c}50.0^{\circ} \\
\pm 30\end{array}$ & $\begin{array}{l}42.0^{3} \\
\pm 7.0\end{array}$ & $9.460 *$ \\
\hline
\end{tabular}

T:Tratamiento; Rta:Respuesta (machos espermiando/ machos tratados); Vol:Volumen; Mov:Movilidad masal; TA Tiempo de activación; sptz:espermatozoides. *Sólo se presenta un valor debido a que sólo un macho emitió cantidad insuficiente de semen para realizar medición.

además, el porcentaje de movilidad masal también fue mayor en el semen de los machos de T1, T2 y T3 con respecto al grupo control. Por su parte, el tiempo de activación fue igual en todos los tratamientos, presentando un valor mínimo de $42.0 \pm 7.0$ seg (grupo control) y máximo de $64.9 \pm 2.5$ seg (T3). La concentración espermática, fue mayor en los machos del T3 $(p<0.05)$, registrándose un valor de $6.303 \pm 1.025$. espermatozoides $\times 10^{3} / \mu$ l. En el caso del control, sólo se muestra un valor de concentración espermática, puesto que solamente un individuo emitió la cantidad suficiente de semen para realizar esta medición.

En cuanto a los parámetros de movilidad individual, la MTI fue significativamente mayor $(p<0.05)$ en T3 $(88.9 \pm 4.5 \%)$, cuando se comparó con T1 (60.6 $\pm 5.6 \%)$ y sin diferencias significativas con la obtenida en T2 $(68.2 \pm 11.5 \%)$ y en el grupo control ( $81.2 \pm 1.6 \%)$ (Figura 1$)$. La movilidad local y circular no presentaron diferencias significativas entre los diferentes tratamientos ni con el control, obteniéndose valores mínimos y máximos en MTL de $8.0 \pm 2.6$ (T3) y $16.0 \pm 1.6 \%(\mathrm{~T} 1)$ y en MCI de 0 (T3 y Control) y $1.7 \%$ (T1) de MCI (T1), respectivamente (Figura1).

La MPLR, MPLL, MLC no presentaron 


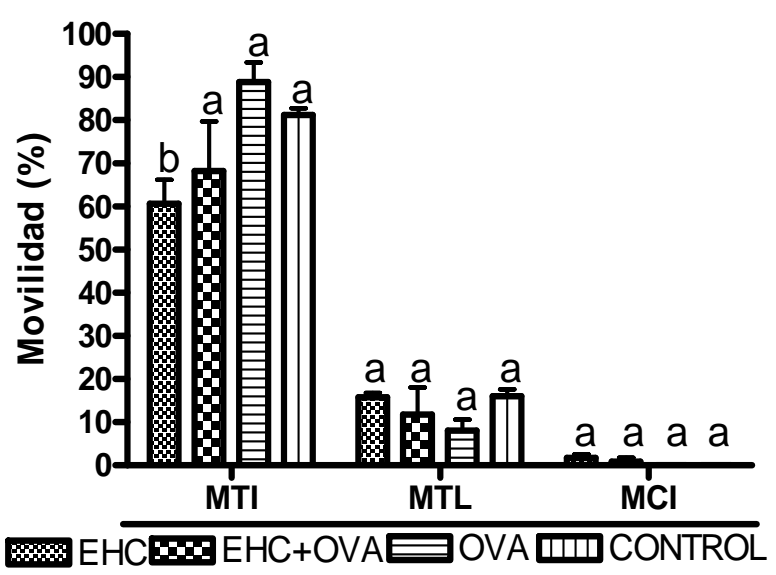

Figura 1. Movilidad espermática individual total, local y circular de yaque, observada después de la inducción de la espermiación con diferentes protocolos hormonales. Letras diferentes indican diferencias significativas $(p<0.05)$. MTI:Movilidad Total, MTL:Movilidad Local, MCI:Movilidad Circular, EHC:Extracto de Hipófisis de Carpa (Tratamiento 1), EHC+OVA:Extracto de Hipófisis de Carpa+Ovaprim (Tratamiento 2), OVA:Ovaprim (Tratamiento 3).

diferencias significativas entre tratamientos ni con el grupo control, observándose valores de $32.4 \pm 6.3$ a $60.1 \pm 13.1 \%$ para MPLR (T2 y T3), de $25.0 \pm 1.0$ a $35.8 \pm 6.8 \%$ para MPLL (T1 y $\mathrm{T} 2$ ) y de $8.0 \pm 2.6$ a $17.5 \pm 0.2 \%$ (T3 y T1), para MLC (Figura 2). Con relación a la IMV, se observaron mayores porcentajes en el T1 $(21.7 \pm 5.7 \%)$ y T2 $(18.9 \pm 5.3 \%)$ que en T3 $(2.9 \pm 2.2 \%)$ y el grupo control

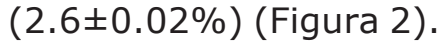

La VCL fue significativamente mayor en el T3 $(64.3 \pm 9.2 \mu \mathrm{m} / \mathrm{seg})$ que en el T1 $(28.5 \pm 2.8)$ y T2 $(34.9 \pm 7.5 \mu \mathrm{m} / \mathrm{seg})$ y $\sin$ diferencias significativas con la del grupo control $(61.8 \pm 2.7 \mu \mathrm{m} / \mathrm{seg})$. La VLR no presentó diferencias significativas entre los tratamientos y el grupo control, con valores de $24.8 \pm 3.3$ a $50.8 \pm 10 \mu \mathrm{m} / \mathrm{seg}$ (T1 y T3). La VPD fue significativamente mayor en T3 $(57.0 \pm 9.6 \mu \mathrm{m} / \mathrm{seg})$ que en T1 $(25.3 \pm 2.9 \mu \mathrm{m} /$ seg) y no presentó diferencias significativas con T2 y el grupo control $(30.3 \pm 8.7$ y $53.5 \pm 3.8 \mu \mathrm{m} / \mathrm{seg}$ )(Figura 3 ).

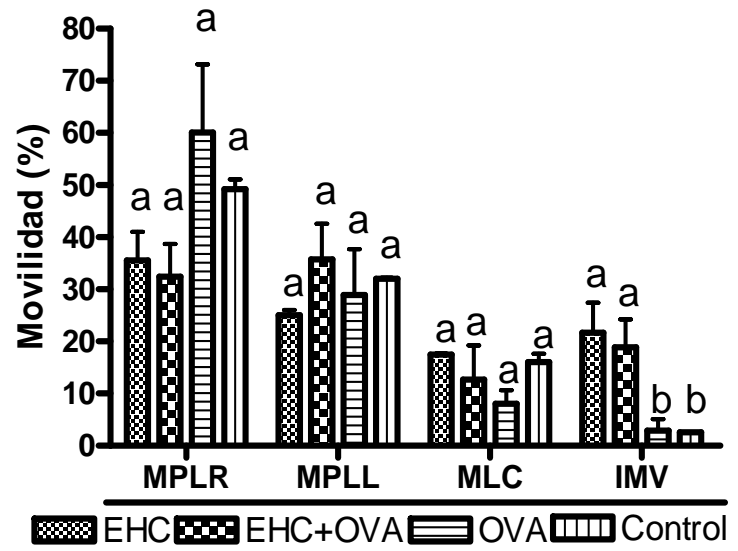

Figura 2. Movilidad espermática individual lineal rápida y lenta, local y porcentaje de espermatozoides inmóviles, de yaque obtenidas con diferentes protocolos de inducción hormonal. Letras diferentes indican diferencias significativas $(p<0.05)$. MPLR:Movilidad progresiva lineal rápida, MPLL:Movilidad progresiva lineal lenta, MLC:Movilidad local o nado en círculo, IMV: Espermatozoides Inmóviles, EHC:Extracto de Hipófisis de Carpa (Tratamiento 1), EHC + OVA:Extracto de Hipófisis de Carpa + Ovaprim (Tratamiento 2), OVA:Ovaprim (Tratamiento 3).

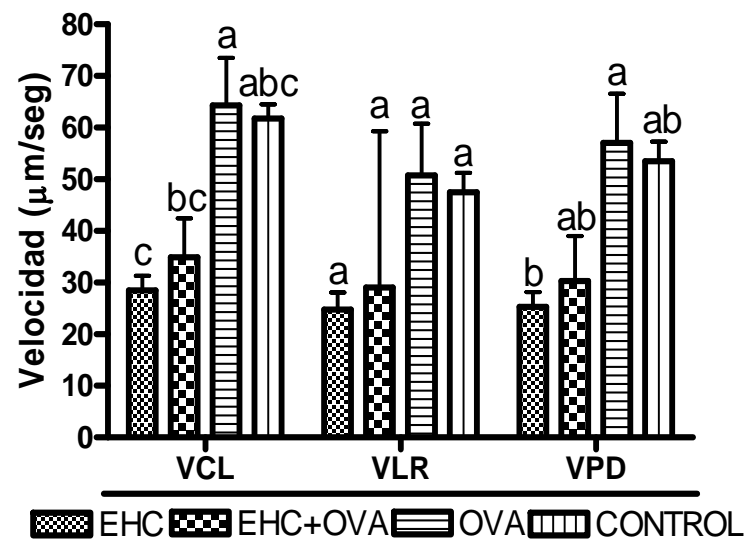

Figura 3. Parámetros de velocidad espermática individual de yaque obtenidos con tres tratamientos hormonales de inducción a la espermiación. Letras diferentes indican diferencias significativas $(p<0.05)$. VCL: Velocidad curvilínea, VLR: Velocidad en línea recta, VPD: Velocidad promedio de desplazamiento, EHC: Extracto de Hipófisis de Carpa (Tratamiento 1), EHC + OVA: Extracto de Hipófisis de Carpa + Ovaprim (Tratamiento 2), OVA: Ovaprim (Tratamiento 3). 


\section{DISCUSIÓN}

En general, los machos de las diferentes especies de peces no necesitan de tratamiento hormonal para inducir la espermiación, no obstante, este proceso optimiza el manejo del semen porque disminuye su viscosidad, lo cual facilita los procesos de fertilización artificial y crioconservación seminal. Sin embargo, en algunas especies de silúridos, como Clarias gariepinus $(2,8)$, Ictalurus punctatus (9), Zungaro jahu (10) y el Leiarius marmoratus (3), no hay liberación espontánea de semen, aún después del tratamiento de inducción hormonal, siendo necesario el sacrificio de los machos para obtener el semen. La obtención del semen a partir del sacrificio de los machos, compromete los intentos de selección y mejoramiento genético de estas especies y es importante lograr disminuir esta práctica para conseguir programas de reproducción económicamente viables $(2,11)$.

Los protocolos evaluados en este trabajo permitieron obtener volúmenes de semen por medio de estrujamiento, adecuados para realizar con éxito procesos de fertilización artificial de oocitos y obtención de alevinos de la especie, sin necesidad de sacrificar los machos para obtener los gametos. El volumen obtenido con los tratamientos evaluados (máximo de $2.84 \mathrm{ml}$ ), fue mayor que el observado por Solano y Urueña (12), quienes evaluaron en L. marmoratus un protocolo consistente en la administración de una dosis única de de EHC (1 mg/ $\mathrm{kg})$, obteniendo $1.9 \pm 1.1 \mathrm{ml}$ de semen. Trabajos realizados en la misma especie, evaluando protocolos con $6 \mathrm{mg} / \mathrm{kg}$ de EHC y $30 \mu \mathrm{g}$ de LHRHa, no lograron obtener semen por estrujamiento y fue necesario extraerlo directamente del testículo por maceración $(3,13)$.

La movilidad espermática es un criterio ampliamente utilizado para evaluar la calidad seminal y es comúnmente utilizado para comparar diferentes condiciones experimentales $(14,15)$. Puede ser evaluada de diversas formas, siendo la más común la observación directa del porcentaje de espermatozoides móviles en un microscopio óptico, usando baja magnificación (movilidad masal). Su estimación es rápida pero presenta discrepancias debido posiblemente a la densidad espermática, que dependiendo si las células observadas son muchas o muy pocas, el porcentaje de movilidad puede resultar sobrevalorado o subvalorado (14). En este estudio, los tratamientos utilizados aumentaron significativamente la movilidad espermática y no afectaron su duración. Los valores hallados en los machos tratados con EHC $(81.3 \pm 4.4 \%)$ fueron superiores a los encontrados en la misma especie con una dosificación menor de EHC (40 $\pm 34 \%)(12)$ y fueron similares a los encontrados en Pseudoplatystoma sp. con el mismo inductor en dosis única $(95 \%)(5,16)$. En Clarias gariepinus no se obtuvo semen por medio de estrujamiento manual cuando se indujo con $8 \mathrm{mg} / \mathrm{kg}$ de EHC; en cambio, cuando se utilizó OVA, hubo liberación de semen pero sin movilidad y cuando se administró una mezcla de EHC y OVA se obtuvo semen pero con movilidad espermática baja (2).

Así como en muchas otras especies, la concentración de los espermatozoides en el fluido seminal también ha sido utilizada tradicionalmente para evaluar la calidad del semen en peces (15). Es conocido que la inducción hormonal aumenta la fluidez del semen disminuyendo la concentración espermática (15), tal como se observó en yaque, donde el número de espermatozoides disminuyó a medida que aumentó el volumen seminal, observándose mayor concentración en los machos tratados con OVA que en aquellos que recibieron EHC o EHC+OVA. Sin embargo, los valores hallados en este trabajo fueron muy similares a los reportados por Solano y Urueña (12), quienes no encontraron efectos de los tratamientos evaluados sobre la cantidad de espermatozoides colectados. En el bagre europeo Silurus glanis, cuando se indujo con EHC se obtuvo mayor número de espermatozoides que cuando se utilizó un implante de un análogo de GnRH (17) y en el bagre rayado Pseudoplatystoma $\mathrm{sp}$. ninguna de las sustancias inductoras afectó la concentración espermática (16).

Recientemente se han introducido nuevas técnicas asistidas por computador (CASA: 
Computer-assisted sperm analysis) que permiten una evaluación de la calidad seminal más objetiva, analizando simultáneamente diferentes parámetros de movilidad y velocidad espermáticas $(14,15)$. A pesar de ser la metodología cuantitativa con más repetibilidad disponible en la actualidad, su utilización es reducida por los altos costos que representa el valor de los equipos utilizados (18), razón por la cual la bibliografía referente a su uso en acuicultura es aún muy limitada. Contrario a lo hallado con la evaluación subjetiva, con la evaluación por medio del CASA se encontró una MTI en el grupo control, T2 y T3, mayor que en T1. En Pseudoplatystoma sp., los tratamientos con EHC y OVA no influyeron en la MTI, mientras que la MTL fue mayor en los tratamientos que en el grupo control (16).

La MTL y la MCI no fueron afectadas por los tratamientos en este trabajo y fueron mayores a las observadas en bagre rayado donde la MTL fue menor con EHC, cuando se comparó con el control (16). La MPLR, MPLL y la MLC tampoco se vieron afectadas por los tratamientos evaluados y el porcentaje de espermatozoides inmóviles fue mayor en T1 y T2 que en T3 y que en el grupo control. En bagre rayado la MPLR y el IMV tampoco fue afectada por los tratamientos, mientras que la MPLL fue menor con EHC que en el grupo control (16). La VCL, VLR y VPD fueron altamente correlacionadas con las tasas de eclosión obtenidas de la fertilización de Clarias gariepinus, usando una relación espermatozoides:oocitos mínima (19). En yaque se obtuvieron valores de VCL y VPD superiores en el tratamiento con OVA a los obtenidos con EHC, mientras que la VLR no fue afectada por los tratamientos. En bagre rayado la VCL, VLR y la VPD fueron mayores en el tratamiento con ovaprim que en el control (16).

En conclusión, el uso de inductores hormonales puede ser efectivo para la liberación de semen en el yaque en cantidades apropiadas para su evaluación y manejo y con características de calidad como movilidad y velocidad espermática, que permitirían obtener tasas de fertilidad satisfactorias en la reproducción de la especie bajo condiciones de cautiverio.

\section{REFERENCIAS}

1. Zohar Y, Mylonas Constantinos C. Endocrine manipulations of spawning in cultured fish: from hormones to genes. Aquaculture 2001;197:99-136.

2. Viveiros ATM, Fessehaye $Y$, ter Veld $M$, Schulz RW, Komen J. Hand-stripping of semen and semen quality after maturational hormone treatments, in African catfish Clarias gariepinus. Aquaculture 2002;213:373-86.

3. Castillo Jiménez AM, Ramírez Lesmes $R$, Rodríguez Pulido JA. Ensayos de reproducción y alevinaje en Yaque Leiarius marmoratus (Gill, 1870) (Pises:Siluriformes:Pimelodidae) en la Orinoquia Colombiana. En: VI Seminario Internacional de Acuicultura I Congreso Nacional de Investigaciones Acuícolas. Bogotá: Universidad Nacional de Colombia, 2003.
4. Muñoz Buelvas RJ, Martínez Díaz CS. Evaluación de la reproducción inducida del blanquillo (Sorubim cuspicudus LITMANN, BURR \& NASS, 2000) con ovaprim ${ }^{\circledR}$. Rev MVZ Córdoba 2003; 8(2):333-334.

5. Guarnizo Pineda M. Caracterización seminal y ensayos preliminares de crioconservación de semen de bagre rayado (Pseudoplatystoma fasciatum - Linnaeus 1766). [Trabajo de Grado]. Palmira, Colombia: Universidad Nacional de Colombia; 2003.

6. Velasco Santamaría YM, Arias Castellanos JA, Cruz Casallas PE. Efecto de la inducción hormonal con extracto de hipófisis de carpa (EHC) sobre algunas características seminales de Rhamdia sebae c.f. En: II Congreso Colombiano de Acuicultura $X$ Jornada de Acuicultura IALL. Villavicencio: Universidad de los Llanos; 2004. 
7. Cacot $P$, Eeckhoutte $P$, Muon DT, Viet Trieu $\mathrm{N}$, Legendre $\mathrm{M}$, Mariojouls $\mathrm{C}$, et al. Induced spermiation and milt management in Pangasius bocourti (Sauvage, 1880). Aquaculture 2003;215:67-77.

8. Brzuska E. Artificial propagation of African catfish (Clarias gariepinus): differences between reproduction effects after stimulation of ovulation with carp pituitary homogenate or $\mathrm{GnRH}-\mathrm{a}$ and dopaminergic inhibitor. Czech J Anim Sci 2003;48(5):18190.

9. Christensen JM, Tiersch TR. Cryopreservation of channel catfish sperm: effects of cryoprotectant exposure time, cooling rate, thawing conditions, and maleto-male variation. Theriogenology 2005;63:2103-12.

10. Viveiros ATM, Godinho HP. Sperm quality and criopreservation of Brazilian freshwater fish species: a review. Fish Physiol Biochem 2009;35(1):137-50.

11. Chowdhury I, Joy KP. Seminal vesicle and testis secretions in Heteropneustes fossilis (Bloch): composition and effects on sperm motility and fertilization. Aquaculture 2001;193:355-71.

12. Solano Camelo MF, Urueña Bermeo FR. Evaluación comparativa del efecto del extracto pituitario de carpa (EPC) y gonadotropina coriónica humana (HCG) en la espermiación del yaque (Leiarius marmoratus). En: VI Seminario Internacional de Acuicultura. Bogotá: Rev Med Vet Zoot 2007. p. 244.

13. Mora JA, Kossowski C. Reproducción inducida del bagre yaque, Leiarius marmoratus, (Gil, 1870) con aplicación de LHRHa. En: XIII Congreso Venezolano de Producción e Industria Animal. Guarico, Universidad Nacional Experimental de los Llanos Centrales Rómulo Gallegos; 2006.
14. Bobe J, Labbé C. Egg and sperm quality in fish. Gen Comp Endocrinol 2009; 165(3):535-48.

15. Rurangwa E, Kime DE, Ollevier F, Nash JP. The measurement of sperm motility and factors affecting sperm quality in cultured fish. Aquaculture 2004;234:1-28.

16. Rivera López EA. Evaluación de las características del semen de bagre rayado Pseudoplatystoma sp. (PISCES:SILURIDAE) obtenido con diferentes inductores hormonales.[Trabajo de Grado]. Fusagasugá, Colombia: Universidad de Cundinamarca; 2009.

17. Linhart O, Billard R. Spermiation and sperm quality of European catfish (Silurus glanis) after implantation of GnRH analogs and injection of carp pituitary extract. J Appl Ichthyol 1994;10:182-8.

18. Wilson Leedy JG, Ingermann RL. Development of a novel CASA system based on open source software for characterization of zebrafish sperm motility parameters. Theriogenology 2007; 67:661-72.

19. Rurangwa E, Volckaert FAM, Huyskens G, Kime DE, Ollevier F. Quality control of refrigerated and cryopreserved semen using computer-assisted sperm analysis (CASA), viable staining and standardized fertilization in african catfish (Clarias gariepinus). Theriogenology 2001;55:751-69. 